Historic, Archive Document

Do not assume content reflects current

scientific knowledge, policies, or practices. 


$$
\text { \} }
$$




\title{
RETAIL PRICE LIST
}

\author{
-of the-
}

\section{Hillside Nursery, Atlantic, Iowa}

\author{
Roy L. Fancolly, Proprietor
}

One Mile East of Courthouse

Musson Telephone 2 on 19

When Trees and Plants Leave Our Possession, Our Responsibility Ceases

\section{Small Fruits}

Strawberries

Strawberry plants, Spring fruiting, $\$ 1.00$ per 100 Varieties, Senator Dunlap, Warfield, Bederwood, and Lovett.

Progressive, Fall or Everbearing, ....\$2.00 per 100

\section{Raspberries}

Cumberland and Gregg, black caps, 50c a dozen $\$ 3.00$ per 100 .

Cardinal, red,

St. Regis Everbearing $50 \mathrm{c}$ per dozen Snyder Blackberries ... $\$ 1.00$ per dozen $.50 \mathrm{c}$ per dozen

Red and White Currants, 10c each, $\$ 1.00$ dozen Champion Gooseberries, $15 \mathrm{c}$ each, $\$ 1.50$ per doz.

\section{Grapevines}

Concord and Moore's Early (black), Pocklinton (white) $10 \mathrm{c}$ each, $\$ 1.00$ per dozen.

\section{Fruit Trees}

\section{Apple, 50c each}

Varieties: Summer-Red Astrachan, Duchess, Red June, Yellow Transparent. Fall-Wealthy, Fameuse or Snow, Whitney. Winter-Jonathan, Grimes Golden, Delicious, Janet, Stayman, Winesap, Gano, McIntosh Red, Roman Stem.

\section{Cherry, 75c each}

Varieties: Early Richmond, early, and Large Montomerency, about ten days later.

\section{Plum, 50c each}

Varieties: De Soto, Terry, Blue Damson, and the Hansen Hybrids, Opata, Sapa and Waneta.

Champion Early Peach, 50c each

$\checkmark$ Keiffer Pear, 50 c each

Shade Trees

Bechtol's Flowering Crab, 3 to $4 \mathrm{ft}$......... 75c each American Elm, 6 to $8 \mathrm{ft}$. ............................50 each Schweidler's Purple Leaved Maple, 5 to $6 \mathrm{ft}$. Sugar Maple, 8 to $10 \mathrm{ft}$.
Weir's Cut Leaved Maple, 6 to $8 \mathrm{ft}$., .........75c each Russian Olive, 4 to $5 \mathrm{ft}$.,..............................50 each Carolina Poplar, 8 to $10 \mathrm{ft}$............................. 50 each Cut Leaved Weeping Birch, 6 to $8 \mathrm{ft}$....\$1.00 each Niobe or Weeping Willow, 4 to $5 \mathrm{ft}$............35 each

\section{Ornamental Shrubs, 35c each}

Pink Almonds, Purple Fringe or Smoke Tree, Pink Tartarian Honeysuckle, Hydragea, Purple and White Lilacs, Snowballs, Japanese Snowberry, Syringa, Thunberg's Barberry.

\section{Roses, 50c each}

Paul Neyron, Ulrich Brunner (both monthly roses) Madam Plantier (white bush rose), Crimson Rambler and Dorothy Perkins (pink climber)

\section{Miscellaneous}

Red and White Paeonies ........................... 25c each Bleeding Hearts...............................................35 each Ampelopsis Veitcheii or Boston Ivy ........50 each Clematis Jackmanni (purple) ....................50c each Clematis Paniculati (white) ..................... 35c each Hall's Japanese Honeysuckle ......................25c each

\section{Ask for prices on Evergreens}

\section{Forest Tree Seedlings}

If we receive the order before April 1 we can furnish forest tree seedlings at the following rates:

American Ash, 12 to 18 inch, Catalpa Speciosa, 18 to 24 inch, American Elm, 18 to 24 inch at $\$ 1.50$ per 100. Russian Mulberry, 18 to 24 in. at $\$ 1.75$ per 100 and Carolina Poplar, 2 to $3 \mathrm{ft}$. at $\$ 3.00$ per 100 .

\section{Asparagus Roots, 25c per dozen}

Rhubarb, divided roots, $50 \mathrm{c}$ per dozen

\section{Vegetable Plants}

During the season, we can furnish vegetable plants as follows: Early cabbage ready about April 15, Tomato ready about May 10, Late Cabbage ready about June 1. Also Pepper, Egg Plant, Cauliflower, Celery and Sweet Potato. 
The Hillside Nursery

Roy L. Fancolly, Prop.

Atlantic, Iowa 